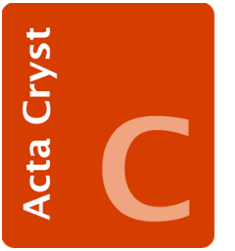

STRUCTURAL

CHEMISTRY

Volume 74 (2018)

Supporting information for article:

Chiral one-dimensional hydrogen-bonded architectures constructed from single-enantiomer phosphoric triamides

Mahsa Eghbali Toularoud, Mehrdad Pourayoubi, Michal Dušek, Václav Eigner and Krishnan Damodaran 
Table S1 Contribution of close contacts in fingerprint plots for L-1 and D-1.

\begin{tabular}{lllll}
\hline & L-1a (\%) & L-1b (\%) & D-1a (\%) & D-1b (\%) \\
H...H & 51.8 & 53.9 & 51.6 & 53.7 \\
C...H/H... & 21.8 & 20.5 & 21.9 & 20.7 \\
F...H/H... & 11.3 & 11.5 & 11.3 & 11.5 \\
O...H/H... & 9.2 & 9.2 & 9.2 & 9.3 \\
Others & 5.9 & 4.9 & 5.4 & 4.9 \\
\hline
\end{tabular}

$\mathrm{a}(\mathrm{F} \ldots \mathrm{O} / \mathrm{O} \ldots \mathrm{F})+(\mathrm{F} \ldots \mathrm{C} / \mathrm{C} \ldots \mathrm{F})+(\mathrm{O} \ldots \mathrm{C} / \mathrm{C} \ldots \mathrm{O})+(\mathrm{N} \ldots \mathrm{H} / \mathrm{H} \ldots \mathrm{N})+(\mathrm{C} \ldots \mathrm{C})$.

\section{Table S2}

\begin{tabular}{|c|c|c|c|c|c|c|c|c|}
\hline \multicolumn{4}{|l|}{${ }^{31} \mathbf{P}(\mathbf{L}-1)$} & \multicolumn{5}{|l|}{${ }^{31} \mathbf{P}(\mathrm{D}-1)$} \\
\hline Run 1 & Run 2 & Run 3 & STDEV & Run 1 & Run 2 & Run 3 & STDEV & Atom \\
\hline 3.7780 & 3.7782 & 3.7780 & 0.0001 & 3.7963 & 3.7963 & 3.7963 & 0.0000 & $\mathrm{P} 1$ \\
\hline${ }^{19} \mathrm{~F}(\mathrm{~L}-1)$ & & & & ${ }^{19} \mathrm{~F}(\mathrm{D}-1)$ & & & & \\
\hline-113.4781 & -113.4782 & -113.4781 & 0.0000 & -113.4659 & -113.4659 & -113.4658 & 0.0000 & $\mathrm{~F} 1 / \mathrm{F} 2$ \\
\hline-113.4903 & -113.4904 & -113.4903 & 0.0000 & -113.4781 & -113.4780 & -113.4780 & 0.0000 & $\mathrm{~F} 1 / \mathrm{F} 2$ \\
\hline-113.5027 & -113.5026 & -113.5026 & 0.0000 & -113.4904 & -113.4904 & -113.4903 & 0.0000 & $\mathrm{~F} 1 / \mathrm{F} 2$ \\
\hline${ }^{1} \mathrm{H}(\mathrm{L}-1)$ & & & & ${ }^{1} \mathrm{H}(\mathrm{D}-1)$ & & & & \\
\hline 9.7598 & 9.7595 & 9.7594 & 0.0002 & 9.7779 & 9.7783 & 9.7784 & 0.0002 & $\underline{\mathrm{HN}} 1$ \\
\hline -- & -- & -- & -- & 9.7678 & 9.7683 & 9.7680 & 0.0002 & $\underline{\mathrm{HN}} 1$ \\
\hline 7.5144 & 7.5144 & 7.5144 & 0.0000 & 7.5140 & 7.5139 & 7.5140 & 0.0000 & $\underline{\mathrm{HC}} 5$ \\
\hline 7.5114 & 7.5114 & 7.5114 & 0.0000 & 7.5109 & 7.5109 & 7.5109 & 0.0000 & $\underline{\mathrm{HC}} 5$ \\
\hline 7.5004 & 7.5004 & 7.5004 & 0.0000 & 7.4999 & 7.4999 & 7.4999 & 0.0000 & $\underline{\mathrm{HC}} 5$ \\
\hline 7.4894 & 7.4893 & 7.4893 & 0.0000 & 7.4890 & 7.4889 & 7.4889 & 0.0000 & $\underline{\mathrm{HC}} 5$ \\
\hline 7.4864 & 7.4864 & 7.4864 & 0.0000 & 7.4859 & 7.4859 & 7.4860 & 0.0000 & $\underline{\mathrm{HC}} 5$ \\
\hline 7.3725 & 7.3725 & 7.3725 & 0.0000 & 7.3760 & 7.3760 & 7.3761 & 0.0000 & $\underline{\mathrm{HC}} 11 / \underline{\mathrm{HC}} 15 / \underline{\mathrm{HC}} 19 / \underline{\mathrm{H}} \mathrm{C} 23$ \\
\hline 7.3602 & 7.3602 & 7.3602 & 0.0000 & 7.3638 & 7.3638 & 7.3638 & 0.0000 & $\underline{\mathrm{HC}} 11 / \underline{\mathrm{HC}} 15 / \underline{\mathrm{HC}} 19 / \underline{\mathrm{HC}} 23$ \\
\hline 7.3373 & 7.3373 & 7.3373 & 0.0000 & 7.3412 & 7.3412 & 7.3412 & 0.0000 & $\underline{\mathrm{HC}} 11 / \underline{\mathrm{HC}} 15 / \underline{\mathrm{HC}} 19 / \underline{\mathrm{HC}} 23$ \\
\hline 7.3251 & 7.3251 & 7.3252 & 0.0000 & 7.3291 & 7.3290 & 7.3291 & 0.0000 & $\underline{\mathrm{HC}} 11 / \underline{\mathrm{HC}} 15 / \underline{\mathrm{HC}} 19 / \underline{\mathrm{HC}} 23$ \\
\hline 7.3028 & 7.3027 & 7.3027 & 0.0000 & 7.3048 & 7.3047 & 7.3048 & 0.0000 & $\underline{\mathrm{HC}} 11 / \underline{\mathrm{HC}} 15 / \underline{\mathrm{HC}} 19 / \underline{\mathrm{HC}} 23$ \\
\hline 7.2995 & 7.2995 & 7.2995 & 0.0000 & 7.3016 & 7.3015 & 7.3016 & 0.0000 & $\underline{\mathrm{HC}} 12 / \underline{\mathrm{HC}} 14 / \underline{\mathrm{HC}} 20 / \underline{\mathrm{HC}} 22$ \\
\hline 7.2903 & 7.2903 & 7.2903 & 0.0000 & 7.2923 & 7.2923 & 7.2923 & 0.0000 & $\underline{\mathrm{HC}} 12 / \underline{\mathrm{HC}} 14 / \underline{\mathrm{HC}} 20 / \underline{\mathrm{HC}} 22$ \\
\hline 7.2798 & 7.2797 & 7.2797 & 0.0000 & 7.2819 & 7.2818 & 7.2819 & 0.0000 & $\underline{\mathrm{HC}} 12 / \underline{\mathrm{HC}} 14 / \underline{\mathrm{HC}} 20 / \underline{\mathrm{HC}} 22$ \\
\hline 7.2771 & 7.2771 & 7.2771 & 0.0000 & 7.2792 & 7.2791 & 7.2792 & 0.0000 & $\underline{\mathrm{HC}} 12 / \underline{\mathrm{HC}} 14 / \underline{\mathrm{HC}} 20 / \underline{\mathrm{HC}} 22$ \\
\hline 7.2721 & 7.2721 & 7.2721 & 0.0000 & 7.2743 & 7.2743 & 7.2743 & 0.0000 & $\underline{\mathrm{HC}} 12 / \underline{\mathrm{HC}} 14 / \underline{\mathrm{HC}} 20 / \underline{\mathrm{HC}} 22$ \\
\hline 7.2689 & 7.2689 & 7.2690 & 0.0000 & 7.2712 & 7.2711 & 7.2712 & 0.0000 & $\underline{\mathrm{HC}} 12 / \underline{\mathrm{HC}} 14 / \underline{\mathrm{HC}} 20 / \underline{\mathrm{HC}} 22$ \\
\hline 7.2597 & 7.2597 & 7.2597 & 0.0000 & 7.2620 & 7.2619 & 7.2620 & 0.0000 & $\underline{\mathrm{HC}} 12 / \underline{\mathrm{HC}} 14 / \underline{\mathrm{HC}} 20 / \underline{\mathrm{HC}} 22$ \\
\hline 7.2493 & 7.2493 & 7.2493 & 0.0000 & 7.2516 & 7.2515 & 7.2516 & 0.0000 & $\underline{\mathrm{HC}} 12 / \underline{\mathrm{HC}} 14 / \underline{\mathrm{H}} \mathrm{C} 20 / \underline{\mathrm{H}} \mathrm{C} 22$ \\
\hline
\end{tabular}




\section{Table S2 continue}

\begin{tabular}{|c|c|c|c|c|c|c|c|c|}
\hline 7.2467 & 7.2467 & 7.2467 & 0.0000 & 7.2490 & 7.2489 & 7.2490 & 0.0000 & $\underline{\mathrm{H} C} 12 / \underline{\mathrm{H} C} 14 / \underline{\mathrm{HC}} 20 / \underline{\mathrm{H}} 22$ \\
\hline 7.211 & 7.2110 & 7.2109 & 0.0000 & 7.2129 & 7.2128 & 7.2129 & 0.0000 & $\underline{\mathrm{HC}} 13 / \underline{\mathrm{HC}} 21$ or $\underline{\mathrm{HC}} 4 / \underline{\mathrm{HC}} 6$ \\
\hline 7.2089 & 7.2089 & 7.2089 & 0.0000 & 7.2108 & 7.2107 & 7.2108 & 0.0000 & $\underline{\mathrm{HC}} 13 / \underline{\mathrm{HC}} 21$ or $\underline{\mathrm{HC}} 4 / \underline{\mathrm{H}} \mathrm{C} 6$ \\
\hline 7.2068 & 7.2068 & 7.2068 & 0.0000 & 7.2087 & 7.2086 & 7.2087 & 0.0000 & $\underline{\mathrm{HC}} 13 / \underline{\mathrm{HC}} 21$ or $\underline{\mathrm{HC}} 4 / \underline{\mathrm{H} C} 6$ \\
\hline 7.1995 & 7.1995 & 7.1994 & 0.0000 & 7.2014 & 7.2013 & 7.2014 & 0.0000 & $\underline{\mathrm{HC}} 13 / \underline{\mathrm{HC}} 21$ or $\underline{\mathrm{HC}} 4 / \underline{\mathrm{HC}} 6$ \\
\hline 7.1966 & 7.1966 & 7.1966 & 0.0000 & 7.1984 & 7.1984 & 7.1985 & 0.0000 & $\underline{\mathrm{HC}} 13 / \underline{\mathrm{HC}} 21$ or $\underline{\mathrm{HC}} 4 / \underline{\mathrm{HC}} 6$ \\
\hline 7.1935 & 7.1935 & 7.1935 & 0.0000 & 7.1954 & 7.1954 & 7.1954 & 0.0000 & $\underline{\mathrm{HC}} 13 / \underline{\mathrm{HC}} 21$ or $\underline{\mathrm{HC}} 4 / \underline{\mathrm{HC}} 6$ \\
\hline 7.1913 & 7.1913 & 7.1914 & 0.0000 & 7.1933 & 7.1932 & 7.1933 & 0.0000 & $\underline{\mathrm{HC}} 13 / \underline{\mathrm{HC}} 21$ or $\underline{\mathrm{HC}} 4 / \underline{\mathrm{HC}} 6$ \\
\hline 7.1866 & 7.1866 & 7.1865 & 0.0000 & 7.1885 & 7.1884 & 7.1885 & 0.0000 & $\underline{\mathrm{HC}} 13 / \underline{\mathrm{HC}} 21$ or $\underline{\mathrm{HC}} 4 / \underline{\mathrm{HC}} 6$ \\
\hline 7.1845 & 7.1845 & 7.1845 & 0.0000 & 7.1864 & 7.1863 & 7.1864 & 0.0000 & $\underline{\mathrm{HC}} 13 / \underline{\mathrm{HC}} 21$ or $\underline{\mathrm{HC}} 4 / \underline{\mathrm{HC}} 6$ \\
\hline 7.1814 & 7.1814 & 7.1814 & 0.0000 & 7.1833 & 7.1833 & 7.1833 & 0.0000 & $\underline{\mathrm{HC}} 13 / \underline{\mathrm{HC}} 21$ or $\underline{\mathrm{HC}} 4 / \underline{\mathrm{HC}} 6$ \\
\hline 7.1782 & 7.1782 & 7.1782 & 0.0000 & 7.1801 & 7.1800 & 7.1801 & 0.0000 & $\underline{\mathrm{HC}} 13 / \underline{\mathrm{HC}} 21$ or $\underline{\mathrm{HC}} 4 / \underline{\mathrm{HC}} 6$ \\
\hline 7.1712 & 7.1712 & 7.1712 & 0.0000 & 7.1732 & 7.1731 & 7.1732 & 0.0000 & $\underline{\mathrm{HC}} 13 / \underline{\mathrm{HC}} 21$ or $\underline{\mathrm{HC}} 4 / \underline{\mathrm{HC}} 6$ \\
\hline 7.1692 & 7.1692 & 7.1692 & 0.0000 & 7.1711 & 7.1710 & 7.1711 & 0.0000 & $\underline{\mathrm{HC}} 13 / \underline{\mathrm{HC}} 21$ or $\underline{\mathrm{HC}} 4 / \underline{\mathrm{HC}} 6$ \\
\hline 7.1671 & 7.1670 & 7.1671 & 0.0000 & 7.1689 & 7.1689 & 7.1690 & 0.0000 & $\underline{\mathrm{HC}} 13 / \underline{\mathrm{HC}} 21$ or $\underline{\mathrm{HC}} 4 / \underline{\mathrm{H} C} 6$ \\
\hline 7.1507 & 7.1507 & 7.1507 & 0.0000 & 7.1506 & 7.1506 & 7.1506 & 0.0000 & $\underline{\mathrm{HC}} 13 / \underline{\mathrm{HC}} 21$ or $\underline{\mathrm{HC}} 4 / \underline{\mathrm{HC}} 6$ \\
\hline 7.1372 & 7.1371 & 7.1372 & 0.0000 & 7.1370 & 7.1370 & 7.1371 & 0.0000 & $\underline{\mathrm{HC}} 13 / \underline{\mathrm{HC}} 21$ or $\underline{\mathrm{HC}} 4 / \underline{\mathrm{HC}} 6$ \\
\hline 7.1237 & 7.1237 & 7.1237 & 0.0000 & 7.1236 & 7.1235 & 7.1236 & 0.0000 & $\underline{\mathrm{HC}} 13 / \underline{\mathrm{HC}} 21$ or $\underline{\mathrm{HC}} 4 / \underline{\mathrm{HC}} 6$ \\
\hline 4.9277 & 4.9278 & 4.9278 & 0.0000 & 4.9328 & 4.9328 & 4.9330 & 0.0001 & $\underline{\mathrm{H}} \mathrm{N} 2 / \underline{\mathrm{HN}} 3$ \\
\hline 4.9105 & 4.9106 & 4.9107 & 0.0001 & 4.9156 & 4.9157 & 4.9158 & 0.0001 & $\underline{\mathrm{HN}} 2 / \underline{\mathrm{HN}} 3$ \\
\hline 4.8934 & 4.8933 & 4.8934 & 0.0000 & 4.8984 & 4.8983 & 4.8985 & 0.0001 & $\underline{\mathrm{HN}} 2 / \underline{\mathrm{HN}} 3$ \\
\hline 4.8408 & 4.8408 & 4.8409 & 0.0000 & 4.8468 & 4.8468 & 4.8470 & 0.0001 & $\underline{\mathrm{H}} 2 / \underline{\mathrm{HN}} 3$ \\
\hline 4.8245 & 4.8245 & 4.8246 & 0.0000 & 4.8305 & 4.8305 & 4.8307 & 0.0001 & $\underline{\mathrm{H}} 2 / \underline{\mathrm{HN}} 3$ \\
\hline 4.8077 & 4.8077 & 4.8078 & 0.0000 & 4.8136 & 4.8137 & 4.8138 & 0.0001 & $\underline{\mathrm{HN}} 2 / \underline{\mathrm{HN}} 3$ \\
\hline 4.4063 & 4.4061 & 4.4062 & 0.0001 & 4.4113 & 4.4113 & 4.4114 & 0.0000 & $\underline{\mathrm{HC}} 8 / \underline{\mathrm{HC}} 16$ \\
\hline 4.3947 & 4.3946 & 4.3945 & 0.0001 & 4.3997 & 4.3997 & 4.3997 & 0.0000 & $\underline{\mathrm{HC}} 8 / \underline{\mathrm{HC}} 16$ \\
\hline 4.3552 & 4.3553 & 4.3551 & 0.0001 & 4.3608 & 4.3607 & 4.3607 & 0.0000 & $\underline{\mathrm{HC}} 8 / \underline{\mathrm{HC}} 16$ \\
\hline 4.3437 & 4.3439 & 4.3437 & 0.0001 & 4.3494 & 4.3493 & 4.3494 & 0.0000 & $\underline{\mathrm{HC}} 8 / \underline{\mathrm{HC}} 16$ \\
\hline 3.3070 & 3.3070 & 3.3071 & 0.0000 & 3.3169 & 3.3169 & 3.3171 & 0.0001 & $\mathrm{H}_{2} \mathrm{O}$ \\
\hline 2.5061 & 2.5061 & 2.5061 & 0.0000 & 2.5062 & 2.5061 & 2.5062 & 0.0000 & solvent \\
\hline 2.5031 & 2.5031 & 2.5031 & 0.0000 & 2.5031 & 2.5030 & 2.5031 & 0.0000 & solvent \\
\hline 2.5000 & 2.5000 & 2.5000 & 0.0000 & 2.5000 & 2.5000 & 2.5000 & 0.0000 & solvent \\
\hline 2.4969 & 2.4969 & 2.4969 & 0.0000 & 2.4969 & 2.4969 & 2.4969 & 0.0000 & solvent \\
\hline 2.4938 & 2.4938 & 2.4938 & 0.0000 & 2.4938 & 2.4938 & 2.4938 & 0.0000 & solvent \\
\hline 1.4115 & 1.4115 & 1.4115 & 0.0000 & 1.4151 & 1.4151 & 1.4151 & 0.0000 & $\underline{\mathrm{HC}} 9 / \underline{\mathrm{HC}} 17$ \\
\hline 1.4000 & 1.4000 & 1.4000 & 0.0000 & 1.4036 & 1.4036 & 1.4036 & 0.0000 & $\underline{\mathrm{HC}} 9 / \underline{\mathrm{HC}} 17$ \\
\hline 1.3962 & 1.3962 & 1.3962 & 0.0000 & 1.4009 & 1.4008 & 1.4009 & 0.0000 & $\underline{\mathrm{HC}} 9 / \underline{\mathrm{HC}} 17$ \\
\hline 1.3847 & 1.3847 & 1.3847 & 0.0000 & 1.3893 & 1.3893 & 1.3893 & 0.0000 & $\underline{\mathrm{HC}} 9 / \underline{\mathrm{HC}} 17$ \\
\hline
\end{tabular}




\section{Table S2 continue}

\begin{tabular}{|c|c|c|c|c|c|c|c|c|}
\hline${ }^{13} \mathrm{C}(\mathrm{L}-1)$ & & & & ${ }^{13} \mathrm{C}(\mathrm{D}-1)$ & & & & \\
\hline 161.6624 & 161.6806 & 161.6758 & 0.0077 & 161.6884 & 161.6905 & 161.6902 & 0.0009 & $\mathrm{C} 1$ \\
\hline 159.6516 & 159.6501 & 159.6521 & 0.0008 & 159.6629 & 159.6632 & 159.6640 & 0.0005 & $\mathrm{C} 3 / \mathrm{C} 7$ \\
\hline 159.5991 & 159.6000 & 159.5996 & 0.0004 & 159.6127 & 159.6119 & 159.6126 & 0.0004 & $\mathrm{C} 3 / \mathrm{C} 7$ \\
\hline 158.0022 & 158.0025 & 158.0019 & 0.0002 & 158.0145 & 158.0136 & 158.0144 & 0.0004 & $\mathrm{C} 3 / \mathrm{C} 7$ \\
\hline 157.9509 & 157.9513 & 157.9518 & 0.0004 & 157.9628 & 157.9619 & 157.9620 & 0.0004 & $\mathrm{C} 3 / \mathrm{C} 7$ \\
\hline 146.2603 & 146.2604 & 146.2603 & 0.0000 & 146.2636 & 146.2633 & 146.2635 & 0.0001 & $\mathrm{C} 10 / \mathrm{C} 18$ \\
\hline 146.2236 & 146.2233 & 146.2229 & 0.0003 & 146.2263 & 146.2263 & 146.2262 & 0.0000 & $\mathrm{C} 10 / \mathrm{C} 18$ \\
\hline 145.9081 & 145.9087 & 145.9080 & 0.0003 & 145.9119 & 145.9116 & 145.9113 & 0.0002 & $\mathrm{C} 10 / \mathrm{C} 18$ \\
\hline 145.8706 & 145.8707 & 145.8699 & 0.0004 & 145.8737 & 145.8739 & 145.8737 & 0.0001 & $\mathrm{C} 10 / \mathrm{C} 18$ \\
\hline 131.8322 & 131.8291 & 131.8292 & 0.0015 & 131.8328 & 131.8330 & 131.8372 & 0.0001 & $C 5^{\mathrm{a}}$ \\
\hline 127.9944 & 127.9945 & 127.9941 & 0.0002 & 128.0019 & 128.0019 & 128.0019 & 0.0000 & $\mathrm{C} 12 / \mathrm{C} 14 / \mathrm{C} 20 / \mathrm{C} 22$ \\
\hline 127.9829 & 127.9830 & 127.9827 & 0.0001 & 127.9905 & 127.9905 & 127.9904 & 0.0000 & $\mathrm{C} 12 / \mathrm{C} 14 / \mathrm{C} 20 / \mathrm{C} 22$ \\
\hline 126.3599 & 126.3601 & 126.3596 & 0.0002 & 126.3683 & 126.3683 & 126.3682 & 0.0000 & $\mathrm{C} 13 / \mathrm{C} 21$ \\
\hline 126.3226 & 126.3228 & 126.3223 & 0.0002 & 126.3311 & 126.3310 & 126.3310 & 0.0000 & $\mathrm{C} 13 / \mathrm{C} 21$ \\
\hline 126.0595 & 126.0596 & 126.0592 & 0.0002 & 126.0677 & 126.0678 & 126.0677 & 0.0000 & $\mathrm{C} 11 / \mathrm{C} 15 / \mathrm{C} 19 / \mathrm{C} 23$ \\
\hline 125.9774 & 125.9775 & 125.9771 & 0.0002 & 125.9860 & 125.9860 & 125.9859 & 0.0000 & C11/C15/C19/C23 \\
\hline 111.9114 & 111.9125 & 111.9120 & 0.0004 & 111.9161 & 111.9155 & 111.9155 & 0.0003 & $\mathrm{C} 4 / \mathrm{C} 6$ \\
\hline 111.7507 & 111.7506 & 111.7501 & 0.0003 & 111.7539 & 111.7541 & 111.7531 & 0.0001 & $\mathrm{C} 4 / \mathrm{C} 6$ \\
\hline 50.0446 & 50.0438 & 50.0440 & 0.0003 & 50.0551 & 50.0555 & 50.0552 & 0.0002 & $\mathrm{C} 8 / \mathrm{C} 16$ \\
\hline 49.7068 & 49.7080 & 49.7083 & 0.0006 & 49.7165 & 49.7168 & 49.7166 & 0.0001 & $\mathrm{C} 8 / \mathrm{C} 16$ \\
\hline 25.4627 & 25.4626 & 25.4615 & 0.0005 & 25.4654 & 25.4660 & 25.4655 & 0.0000 & $\mathrm{C} 9 / \mathrm{C} 17$ \\
\hline 25.4277 & 25.4276 & 25.4272 & 0.0002 & 25.4312 & 25.4315 & 25.4311 & 0.0002 & C9/C17 \\
\hline 24.9121 & 24.9115 & 24.9112 & 0.0004 & 24.9131 & 24.9134 & 24.9133 & 0.0001 & C9/C17 \\
\hline 24.8790 & 24.8792 & 24.8782 & 0.0004 & 24.8805 & 24.8808 & 24.8801 & 0.0003 & C9/C17 \\
\hline
\end{tabular}

a The signal around 131.8 are triplet (C5) and the S/N of the triplet wasn't good enough to report reproducibility. So I used just the center signal. Moreover, for the signal about 115.3 (double of triplets, C2), the peak had a very low $\mathrm{S} / \mathrm{N}$ to report reliably.

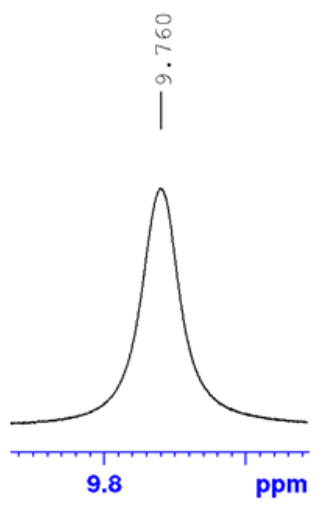

(L-1)

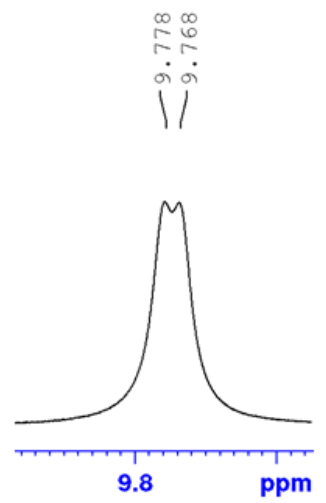

(D-1)

Figure S1 The signal of $\mathrm{N}_{\mathrm{CPH}} \mathrm{H}$ proton for compounds L-1 and D-1. 

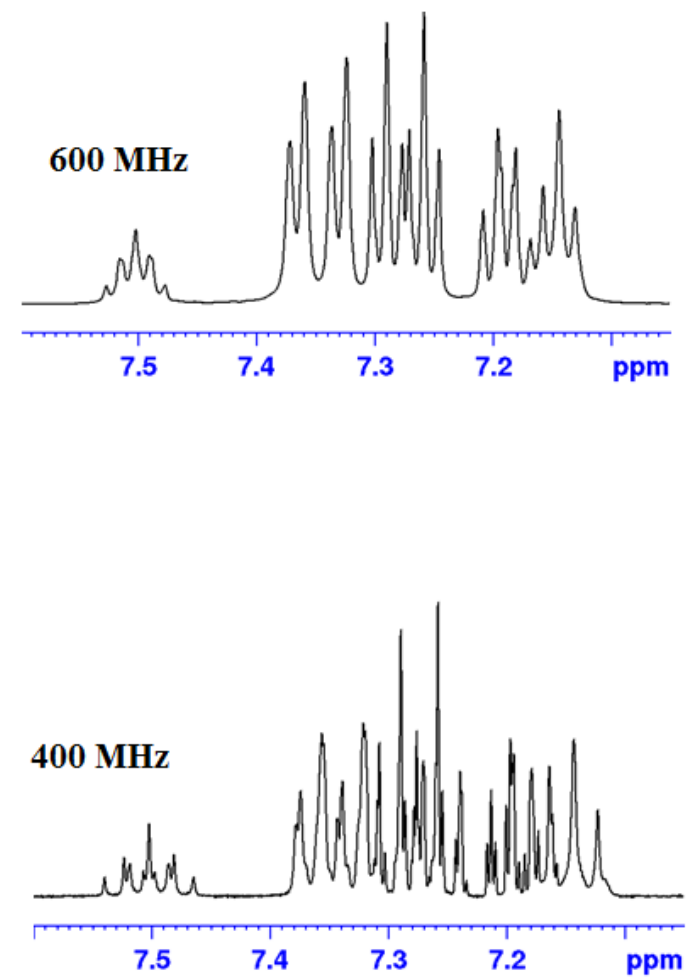

Figure S2 Selected region of ${ }^{1} \mathrm{HNMR}$ spectra at two different frequencies, for L-1 in DMSO- $d_{6}$. 